\title{
Effect of user fee on patient's welfare and efficiency in a two tier health care market
}

\section{Eugenia Amporfu}

Kwame Nkrumah University of Science and Technology, Kumasi, Ghana; eamporfu@gmail.com

Received 26 March 2010; revised 27 May 2010; accepted 29 May 2010.

\begin{abstract}
This is a theoretical paper examining the effect of user fee on patients' welfare and social welfare under three forms of provider reimbursements: full cost, prospective payment and cost sharing. The paper extends Rickman and McGuire (1999) by introducing user fee to the public sector and maintaining the assumption that providers can work in both the private and public health sectors. Contrary to previous studies, this study shows that efficiency is possible under the full cost reimbursement. The paper also shows the conditions under which efficiency is possible under each reimbursement scheme. Patient's welfare can improve with the introduction of user fee when services in the public and private sector are complementary.
\end{abstract}

Keywords: User Fee; Two Tier Healthcare; Mixed Financing; Prospective Payment; Cost Sharing

\section{INTRODUCTION}

Many countries have the two tier health care system: the coexistence of public and private health care sectors. And over the years, public health care reform in many countries has involved a switch from fully financed system to mixed ones $[1,2]$. Such a switch is observed in both industrialized and developing countries. The mixed financing system involves making the patient bear at least part of the cost of care provided in the public sector. The intention is to partly relieve the government of the burden of funding public health care and at the same time reduce excessive use of care that might exist under the fully funded system. Various payment schemes are also used in the public health sector to pay health care providers. The purpose of this paper is to find the impact of patient direct payment at the point of purchase, in the public health sector, on the efficiency of three payment schemes: full cost reimbursement, prospective payment and cost sharing in a two tier health care sector.

Example of mixed financing systems in industrialised countries consists of a combination of compulsory social security system covering a package of essential services and private insurance policy to cover the rest. The patient then has to pay premium and co-payment [3]. Some of the industrialised countries that have adopted this include Australia, Italy, and the United States (in the Medicare plan) [3]. In developing countries the mixed financing system in general involves introduction of user fee. Many Sub-Saharan countries such as Cote d'Ivoire, Kenya, and Nigeria have adopted this system.

There is considerable theoretical literature on the effect of private insurance (with co-payment) on quality of care and the efficient provision of services (e.g., [4-8]. While these analyses centred more on the effect of insurance on quality and efficiency of services than on the reimbursement schemes, others examined the role of different reimbursement schemes in the efficient provision of services [9-17]. The question then is does the effect of mixed financing system on efficiency depend on the type of reimbursement scheme to providers.

To answer the question, the current paper extended a model on a two tier health care system in [16] that examined the effect of the private health care sector on the efficiency of provider reimbursement schemes. The extension involves the introduction of a user fee to the public sector. Reference [16] in turn, was an extension of [18], which examined the effect of reimbursement scheme on the supply of services in the public sector. In their study, the public health care was a fully funded system (e.g., the National Health Services in the Britain) and so the patient did not pay for the services received. Reference [16] extended [18] by including a private sector while maintaining the full funding system in the public sector. The specific interest in the paper then is to examine how their results of the two previous papers would be affected when a user fee is included in the public sector. 
Earlier studies that examined health production in private and public sector with user fee in the public sector include [18-20]. Reference [18] presented an empirical model in which effort was not observable and patients had to choose medical contracts for health care provision from government and mission hospitals in the Cameroun. Reference [20] also presented an empirical study, which examined how the introduction of user fee to the public sector affects quality and accessibility of services. In a theoretical model in which public sector services are covered partly by compulsory social insurance, partly by private insurance and partly by out-of-pocket, [21] examined the conditions for optimal rates of social insurance and private coinsurance. Following [16] the current paper focuses on three forms of provider-reimbursement schemes: full-cost reimbursement, prospective payment and cost sharing.

\section{METHOD}

This is a model of mixed health care market, public and private, that allows physicians to work in both sectors. Let $q$ represent publicly provided health care received by a representative patient and $s$ represent privately provided health care. The patient receives benefit, $B(q, s)$, from treatment and pays for it according to the marginal benefit it provides: $B_{s}()$.$s , for treatment in the private$ sector and $(1-\alpha) B_{q}()$.$q for treatment in the public sector,$ where $0<\alpha<1$ is the fraction of the fee paid by the government. These fees do not have to equal the full cost of treatment. The patient could have partial insurance and bear only part of the cost of treatment. What is important is that the patient bears some cost for treatment in both sectors. $B($.$) has positive marginal products, with$ unique maxima in $q$ and $s, B_{q q}<0, B_{s s}<0$. Treatments, $q$ and $s$, can be complements, $B_{s q}()>$.0 , substitutes, $B_{s q}($. $<0$ or unrelated $B_{s q}()=$.0 . Treatments are complements if, for example, the physician uses the public sector to request the patient to do some tests in the private sector

\footnotetext{
${ }^{1}$ Note that $N_{q}$ can be negative when services are complements and $s$ is very large. This case is not examined.

${ }^{2}$ I do not use the assumption in [16] that patients do not search among alternative physicians but because physicians care about the welfare of patients they do not charge a monopolist price. In the current model, the physician's care for the patient represents his care for ethics of treatment . This prevents the physician from charging monopolist price. As shown in (11) the physician would charge a monopolist price if $U_{N}=$ 0 . This is consistent with [23], where the physician is constrained by patient information. In [23] even though, patients cannot evaluate the marginal benefit from treatment from a given physician they can evaluate the absolute utility upon treatment. Patients can observe the utility of other patients after treatment. If patients of one physician end up with a lower utility on average than others then the physician looses patients.

${ }^{3}$ In order to avoid the implied requirement that the marginal cost in the two sectors be equal, I do not assume constant marginal costs for the provision of treatment as in [16].
}

to help with diagnoses in the public sector. When the physician uses, for example, the private sector to treat an illness that can be treated in the public sector then $q$ and $s$ are substitutes. The patient's net benefit is:

$$
N(q, s)=B(q, s)-B_{s}(q, s) s-(1-\alpha) B_{q}(q, s) q
$$

With the exception of the last term on the RHS, which represents the fee in the public sector, (1) is identical to the patient's net benefit in [16]. The marginal net benefits are:

$$
\begin{gathered}
N_{q}(q, s)=\alpha B_{q}(q, s)-q(1-\alpha) B_{q q}(q, s)-s B_{s q}(q, s)>0{ }^{1} \\
N_{s}(q, s)=-s B_{s s}(q, s)-(1-\alpha) B_{q s}(q, s) q>0
\end{gathered}
$$

Physicians are regulated and are often expected to follow a code of ethics with the purpose of taking the patient's interest into account when choosing treatment. Following [22] it is assumed that the physician cares about the ethics of treatment ${ }^{2}$. Thus, the physician cares about the well-being of his patient, $N(q, \mathrm{~s})$, as well as the profit of his public hospital, $\pi^{h}$ and private sector profit, $\pi^{p}$ :

$$
\pi^{h}=R(q)+(1-\alpha) B_{q}(q, s) q-c(q)
$$

where $R(q)$ is the revenue that the public hospital receives from the government. Again with the exception of $(1-\alpha) B_{q}(q, s) q(4)$ is identical to the public hospital profit in [16]. The private profit, however, is the same as that in [16]:

$$
\pi^{p}=B_{s}(q, s) s-c(s)
$$

where $c^{\prime}(\mathrm{i})>0, c^{\prime \prime}(\mathrm{i})>0(\mathrm{i}=q, s),{ }^{3}$ and $\pi^{h}$ and $\pi^{p}$ have unique maximum in $q$ and $s$ respectively, the marginal profits are:

$$
\begin{gathered}
\pi_{s}^{h}=(1-\alpha) B_{s q}(q, s) q \geq \text { or }<0 \\
\pi_{q}^{h}=R^{\prime}(q)+(1-\alpha) B_{q}(q, s) \\
+q B_{q q}(q, s)-c^{\prime}(q) \leq \text { or }>0
\end{gathered}
$$

where $R^{\prime}(q)>0$.

$$
\begin{gathered}
\quad \geq \\
\pi_{q}^{p}=B_{s q}(q, s) s<0 \\
\pi_{s}^{p}=B_{s}(q, s)+s B_{s s}(q, s)-c^{\prime}(s) \geq 0
\end{gathered}
$$

The physician's utility function is $U\left(\pi^{h}, \pi^{p}, N\right)$, with $U_{N}>0, U_{\pi^{h}}>0, U_{\pi^{p}}>0, U_{N N}<0, U_{\pi^{h} \pi^{h}}<0$, and $U_{\pi^{P} \pi^{P}}<0$. The physician chooses $q$ and $s$ to maximize his utility. The first order conditions are:

$$
\begin{aligned}
& U_{\pi^{h}} \pi_{q}^{h}+U_{\pi^{p}} \pi_{q}^{p}+U_{N} N_{q}=0 \\
& U_{\pi^{h}} \pi_{s}^{h}+U_{\pi^{p}} \pi_{s}^{p}+U_{N} N_{s}=0
\end{aligned}
$$




$$
\Rightarrow U_{\pi^{p}}=-\frac{U_{\pi^{n}} \pi_{s}^{h}+U_{N} N_{s}}{\pi_{s}^{p}}
$$

By substituting (12) into (10) and rearranging produces:

$$
\pi_{q}^{h}=M R S_{\pi^{h} N} N_{s}\left(\frac{\pi_{q}^{p}}{\pi_{s}^{p}}-\frac{N_{q}}{N_{s}}\right)+\frac{\pi_{s}^{h} \pi_{q}^{p}}{\pi_{s}^{p}}
$$

where $M R S_{N \pi}^{h}=U_{N} / U \pi^{h}>0$. Eq.13 defines a locus $\{q, s\}$ that maximize the physician's utility. With the exception of the second term on the RHS, (13) is identical to what [16] obtained. This second term appears here because the inclusion of a fee in the public sector makes $\pi_{s}^{h}$, which is zero in [16], positive, negative or zero, in the current model, depending on whether $q$ and $s$ are complements, substitutes, or unrelated respectively. The terms in brackets are the magnitudes of the slopes of the private sector iso-profit $\left(\pi_{q}^{p} / \pi_{s}^{p}\right)$ and the patient's indifference curve $\left(N_{q} / N_{s}\right)$.

The welfare function, $W(q, s)=B(q, s)-c(q)-c(s)$, is used to find efficiency under the various reimbursement schemes. It is assumed that the welfare function is concave and has unique maxima in $q$ and $s$. Efficiency requires that the following first order conditions for the maximization of the welfare function are satisfied:

$$
\begin{aligned}
& B_{q}(q, s)-c^{h}=0 \\
& B_{s}(q, s)-c^{p}=0
\end{aligned}
$$

The three physician reimbursement rules in the public sector and their effect on equilibrium $q$ and $s$ provided by the physician are now examined. The reimbursement rules are full-cost reimbursement, prospective payment, and cost sharing. The patients in [16] did not have to pay fees in the public sector and so the full-cost reimbursement involved the government providing enough revenue to cover the cost of production. In the current model, however, the full-cost reimbursement, involves the public hospital receiving $R(q)$ from the government to cover part of the cost not covered by the user fee. Prospective payment involves the government giving a fixed amount of revenue, $G$, to the hospital regardless of the total cost of production and of the total fees collected. The cost-sharing rule is a combination of prospective payment and cost reimbursement. The government gives fixed revenue, $G$, and then pays for a fraction of the cost of production. Under each rule, (13) is used to examine

\footnotetext{
${ }^{4}$ where $c^{h}$ and $c^{p}$ represent the marginal cost of $q$ and $s$ respectively. ${ }^{5}$ Because the marginal net benefits in this model differ from those in Rickman and McGuire the patient's indifference curves in this model are also different. The indifference curves in Rickman and McGuire slope downward when $q$ and $s$ are substitutes and upward when they are complements (U-shaped indifference curves). In Rickman and McGuire $N_{s}>0$, whether $q$ and $s$ are substitutes or complements; $N_{q}>0$ when they are substitutes and $N_{q}<0$ when they are complements.
}

how the $q$ and $s$ chosen by the physician affect optimality from the points of view of the patient and society.

The patient's indifference curves are downward sloping for both complements and substitutes ${ }^{5}$. This is because the slope of the indifference curve is $-N_{q} / N_{s}$ and with $N_{q}>0, N_{s}>0$ regardless of the relationship between $q$ and $s$. Figures 1(a) and 1(b) shows the iso-profits for the physician's private profit. The slope of the iso-profit $\left(-\pi_{q}^{p} / \pi_{s}^{p}\right)$ depends on whether treatments are substitutes or complements. As shown in (8) and (9), $\pi_{q}^{p}$ is positive when treatments are complements and negative when they are substitutes; $\pi_{s}^{p}$ is positive when $s$ is very small and becomes negative as it is increases. Thus, when treatments are substitutes, the iso-profit slopes upwards when $s$ is small and downwards when $s$ increases with profit increasing as $q$ falls. The opposite occurs when treatments are complements. When treatments are complements the iso-profit slopes downwards when $s$ is small and upwards as $s$ increases with profit increasing in $q$. These are shown in Figure 1.

\section{RESULTS AND DISCUSSION}

\subsection{Full Cost Reimbursement}

Under this rule, the government provides the revenue required to cover part of the cost of production that the user fee could not cover. Hence $\pi^{h}=0$ and so $\pi_{q}^{h}=0$; i.e., the physician chooses $q$ to maximize public hospital profit. Equation (13), then, becomes:

$$
0=M R S_{\pi^{h} N} N_{s}\left(\frac{\pi_{q}^{p}}{\pi_{s}^{p}}-\frac{N_{q}}{N_{s}}\right)+\frac{\pi_{s}^{h} \pi_{q}^{p}}{\pi_{s}^{p}}
$$

Rearranging (16) produces:

$$
\frac{M R S_{N_{\pi^{h}}} N_{s}+\pi_{s}^{h}}{M R S_{N_{\pi^{h}}} N_{s}} \frac{\pi_{q}^{p}}{\pi_{s}^{p}}=\frac{N_{q}}{N_{s}}
$$

Notice that when $\pi_{s}^{h}=0$, the two slopes in brackets in (16) are equal, and the results are identical to those in [16], i.e., the physician chooses $q$ and $s$ to equate the slopes of the iso-profit and the patient's indifference curves. Such result is possible in the current model if $q$ and $s$ are unrelated, i.e., if $B_{q s}(q, s)=0$ implying that $\pi_{s}^{h}$ $=0$. It is of interest to examine the optimality of equilibrium $q$ and $s$ from the patient's and society's perspective when services are complements, $\pi_{s}^{h}>0$, and when they are substitutes, $\pi_{s}^{h}<0$. Eq.17 shows that in equilibrium the difference between the slopes of the isoprofit and indifference curves depends on the sign of $\pi_{s}^{h}$. When treatments are substitutes, $\pi_{s}^{h}<0$, the iso-profit curve is steeper than the patient's indifference curve. The resulting $q$ lowers the patient's welfare compared to [15] as well as [16]. Reference [15] showed that in the ab- 


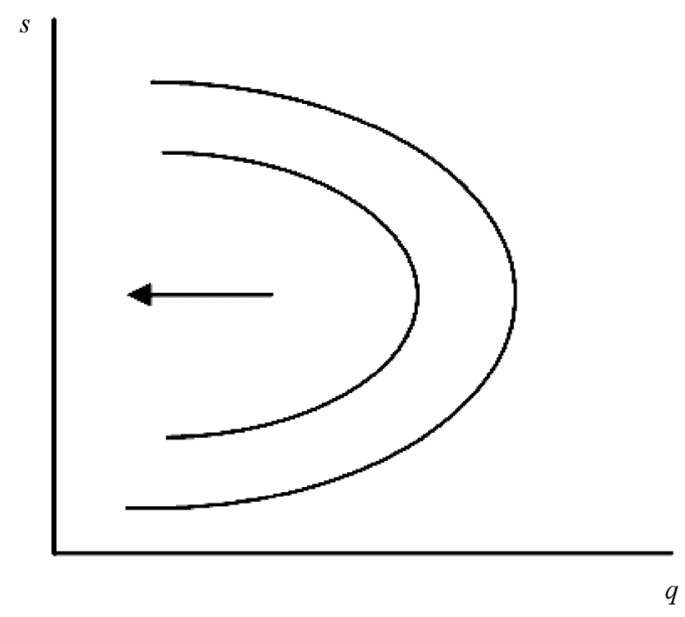

(a)

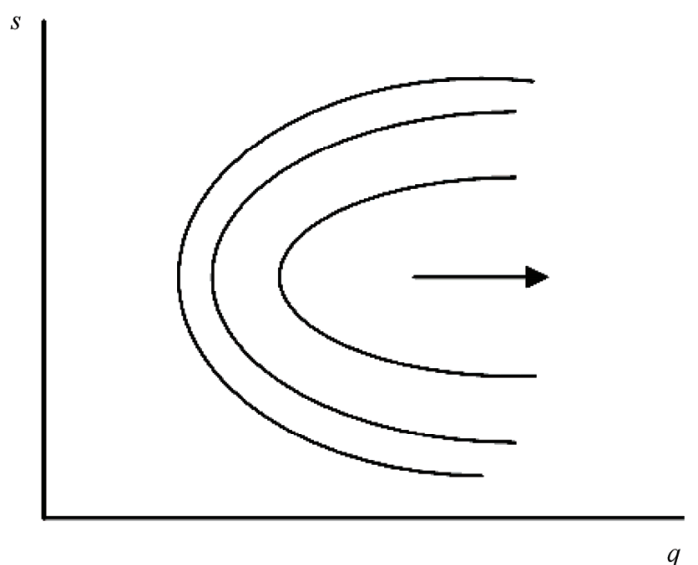

(b)

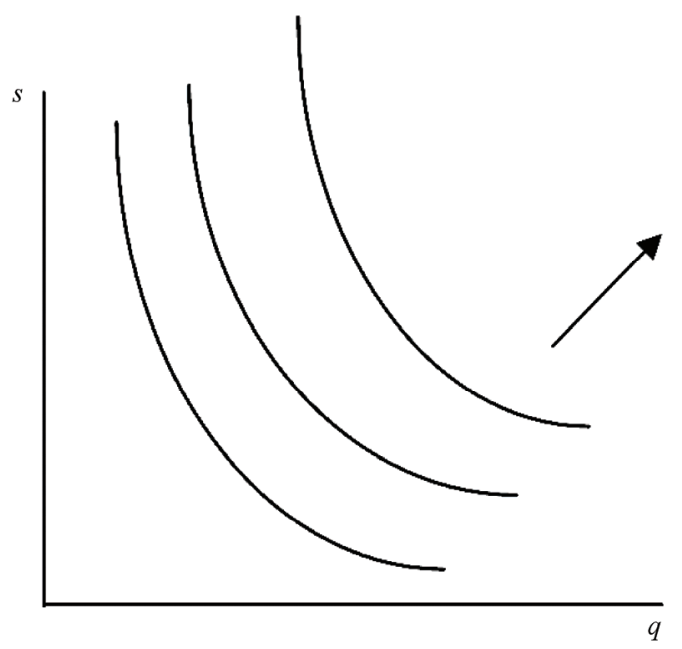

(c)

Figure 1. (a) Private iso-profit curves for substitutes; (b) private iso-profit curves for complements; (c) patient's indifference curves for substitutes and complements. sence of a private sector and a user fee, the optimal $q$ chosen by the physician coincides with what the patient would choose under full information, i.e., at the point where net marginal benefit is zero. However, by including the private sector, [16] showed that the physician reallocates treatments between the private and public sector such that the patient's marginal net benefit in equilibrium is positive; hence, from the patient's perspective, the $q$ chosen by the physician is sub-optimal. In the current model, the inclusion of a user fee further increases the patient's marginal net benefits. The patient, then, gets less $q$ and lower welfare than in [16]. Figure 2(a) shows the equilibrium treatments from each sector when treatments are substitutes.

For complements $N_{s}>0, N_{q}>0$ and $\pi_{s}^{h}>0$ and so in equilibrium the patient's indifference curve is steeper than the iso-profit. The patient, then, receives less supply of both services than he would have chosen himself. This is contrary to [16] where the patient received oversupply of $q$ and undersupply of $s$. It is thus not clear if the patient is worse off or better off in this model than in [16] when services are complements. The equilibrium is shown in Figure 2(b).

The difference between the sign of $\pi_{s}^{h}$ in [16] and the current paper makes an important point. With $\pi_{s}^{h}=0$ in [16], the quantity of $s$ chosen by the physician does not affect the profit of the public hospital. The introduction of user fee in the current paper, however, makes the public hospital profit dependent on the quantity of services supplied in the private sector with public profit decreasing in $s$ when services are substitutes and increasing $s$ when services are complements. When services are substitutes, the two sectors compete for services and so every unit of private treatment supplied represents a loss of fee to the public sector. With complementary services, however, the two sectors become partners and so an increase in supply of private treatment is accompanied by an increase in supply of public treatment and thus increases the amount of fees received by the public hospital. Since the physician is agent to the public as well as the private hospitals, a change in the physician's behaviour that depends on the relationship between the services represents the conflict of interest that exist in the agency relationship. Such conflict of interest can adversely affect the public hospital if the physician puts more weight on private profit than public profit when services are substitutes. When services are complements the two hospitals becomes partners and so any conflict of interest is eliminated. Thus, the standard notion that physician has incentive to create artificial shortages in the public sector in order to increase services in the private sector is relevant when services are substitutes.

The efficiency of the equilibrium is now considered by using (16): 


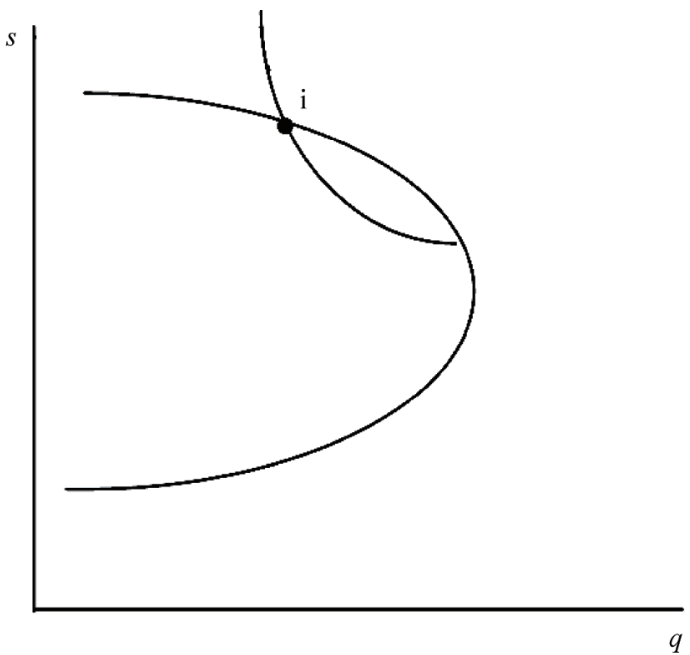

(a)

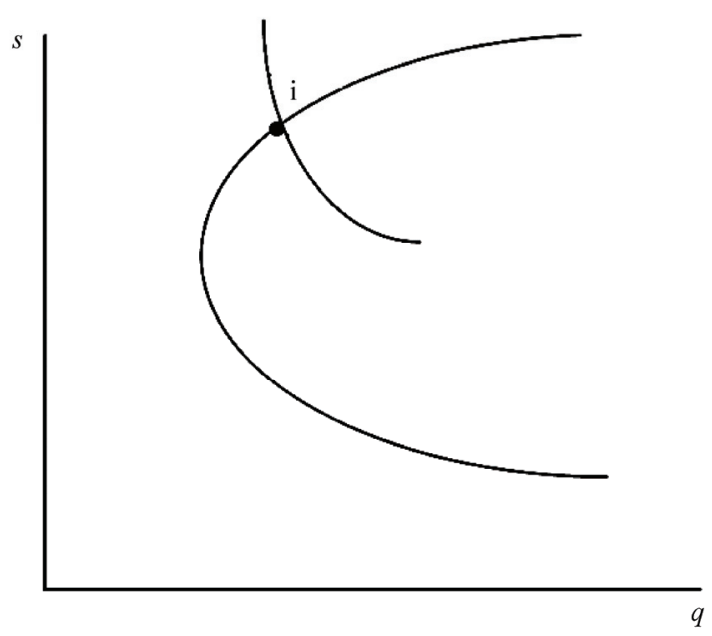

(b)

Figure 2. (a) Full cost equilibrium for substitutes; (b) full cost equilibrium for complements.

$$
0=M R S_{N_{\pi^{h}}} N_{s}\left(\frac{\pi_{q}^{p}}{\pi_{s}^{p}}-\frac{N_{q}}{N_{s}}\right)+\frac{\pi_{s}^{h} \pi_{q}^{p}}{\pi_{s}^{p}}
$$

By substituting (9) into (16) and rearranging (16) becomes:

$$
v=\frac{M R S_{N_{\pi^{h}}} N_{s}+\pi_{s}^{h}}{M R S_{N_{\pi^{h}}} N_{q}} \pi_{q}^{p}-s B_{s s}
$$

where $v=B_{s}-c^{p}$. Assuming efficiency in the private sector, $v=0$, it is of interest to find out if efficiency in the public sector is also achievable. This is done by setting $v=0$ and substituting (2), (3), (6) and (8) into (18)

${ }^{6}$ Note that price elasticity of demand for $q$ is: $\varepsilon_{q}=\frac{B_{q}}{q B_{q q}} \Rightarrow B_{q q}=\frac{B_{q}}{q \varepsilon_{q}}$ and solving for $B_{q}$ :

$$
B_{q}=\frac{(1-\alpha) q B_{q q}+\alpha s B_{s q}-1}{\alpha}+\frac{(1-\alpha) q B_{s q}\left(1-M R S_{N_{\pi^{h}}}\right)}{\alpha s B_{s s} M R S_{N_{\pi^{h}}}}
$$

The sign of $B_{q}$ depends on whether services are complements or substitutes as well as on the size of $M R S_{N \pi} h$. For substitutes, $B_{q}<0<c^{h}$ when $M R S_{N \pi}^{h} \geq 1$ and for complements, $B_{q}<0<c^{h}$ is possible when $M R S_{N \pi}^{h}<1$.

However, $B_{q}>0<c^{h}$ is possible when services are substitutes and $M R S_{N \pi}{ }^{h}<1$ or when services are complements and $M R S_{N \pi}^{h}>1$. For complements when $M R S_{N \pi}^{h}$ $=1, B_{q}<0<c^{h}$ when $q>s$ and demand for $q$ is inelastic ${ }^{6}$. Similarly, $B_{q}>0<c^{h}$ when $q<s$ and demand for $q$ is elastic. $M R S_{N \pi}{ }^{h}=1$, means that the physician puts equal weight on the public hospital's surplus and patient's utility, when choosing treatment. Reference [15], call this behaviour perfect agency. Recall that $M R S_{N \pi}{ }^{h}=U_{N} / U_{\pi}^{h}$, and so $M R S_{N \pi}^{h}>1$ when the physician puts more weight on the patients net benefit than public hospital surplus. Similarly, $M R S_{N \pi}{ }^{h}<1$ when the physician puts more weight on the public hospital surplus than the patient's net benefit. As described in [15], such imperfect agency is likely, because the hospital often has a stronger bargaining power on the physician than the patient. Hence the analysis will focus on the case where $M R S_{N \pi}^{h} \leq 1$. Perfect agent is used to refer to the case in which $M R S_{N \pi}{ }^{h}$ $=1$ while imperfect agent refers to the case in which $M R S_{N \pi}^{h}<1$.

For substitutes, (19) shows that when the supply of $s$ is efficient the physician oversupplies $q$ if he is a perfect agent. In the same way the physician can oversupply $q$ when for complementary services he is an imperfect agent or is a perfect agent, $q>s$, and demand for $q$ is inelastic. For both substitutes and complements efficiency in both sectors can be ruled out under these circumstances. However, efficiency is possible when providing services that are substitutes and the physician is an imperfect agent. When services are complements perfect agency combined with elastic demand for $q$ and a less supply of $q$ than $s$ is required for efficiency.

These results are important in several respects. First, contrary to [16], where efficiency in both sectors is not possible under the full-cost reimbursement rule, efficiency in both sectors is possible in the current model under the full cost. This gives credibility to the argument that cost control policy on the demand-side in the form of user fee, coinsurance, and deductibles is essential to reducing the excessive use of care that exist under the full cost reimbursement scheme with full insurance in the public sector. However, even though efficiency is 
possible it is achieved at the expense of the patient's welfare. This is not surprising because the user fee forces the patient rather than the provider to internalize any externality that existed in the market. As already explained, the user fee reduces the equilibrium supply of services and this makes the patient worse off. Secondly, imperfect agency is required for efficiency. Unlike [16] as well as [15] where the agency role is not able to achieve efficiency under full cost reimbursement, imperfect agency is crucial for efficiency in the current model. In [16], efficiency in the private sector is only achievable at the expense of oversupply of services in the public sector. The possibility of efficiency in the current model then implies that when services are substitutes, the introduction of user fee in the public sector constraints the physician from oversupplying services to the extent of supplying the amount that maximizes social surplus as long as the physician is an imperfect agent. The user fee then transfers any cost caused by the imperfection of the agency unto the patient. Thirdly, the elasticity of demand for public service is important for efficiency when services are complements. For complementary services, the user fee maximizes social surplus by reducing oversupply as long as the physician is a perfect agent when demand for $q$ is elastic and the equilibrium supply of $q$ is less than $s$. When services are complements and there are no close substitutes the resulting inelastic demand for $q$ makes even perfect agency unable to achieve efficiency in the public sector. This weakens the ability of the user fee to achieve the efficiency and so weakens the argument for cost control on the demand side when services are complements.

The results are also contrary to what [24] and [25] found in comparing full cost reimbursement with prospective payment. They show that cost-reimbursement like fee-for-service is characterized by oversupply because it does not provide the provider incentive to economize on the quantity of services. Like [15] they did not have user fee in their models.

\subsection{Prospective Payment}

Under this rule, the government gives a fixed amount of revenue, $G$, to the public hospital regardless of cost of the production and revenue collected from user fee. Thus, (4) becomes:

\footnotetext{
${ }^{7}$ Note Note that a fall in $q$ causes an increase in private profit when services are substitutes. By using the positive sloped portion of the iso-profit it is possible to obtain similar results if the private iso-profit curve is allowed to shift to the left and indifference curve remains unchanged.

${ }^{8}$ The underlying assumption is that $\frac{B_{s q}^{2}-B_{s s} B_{q q}}{B_{s s}}>0$, i.e., the $q$ and $s$ maximize patient benefit if cost were zero, making the term in the square bracket positive.
}

$$
\pi^{h}=G+(1-\alpha) B_{q}(q, s) q-c(q)
$$

The resulting marginal profit with respect to $q$ is negative:

$$
\pi_{q}^{h}=(1-\alpha) B_{q}(q, s)+q(1-\alpha) B_{q q}(q, s)-c^{\prime}(q)<0
$$

Eq.13, then, becomes:

$$
\begin{aligned}
& (1-\alpha) B_{q}(q, s)+q(1-\alpha) B_{q q}(q, s)-c^{\prime}(q) \\
& =M R S_{N_{\pi^{h}}} N_{s}\left(\frac{\pi_{q}^{p}}{\pi_{s}^{p}}-\frac{N_{q}}{N_{s}}\right)+\frac{\pi_{s}^{h} \pi_{q}^{p}}{\pi_{s}^{p}}
\end{aligned}
$$

Rearrange to get:

$$
\begin{aligned}
& \frac{c^{\prime}(q)-q(1-\alpha) B_{q q}(q, s)-(1-\alpha) B_{q}(q, s)}{M R S_{N_{\pi^{h}}} N_{s}} \\
& +\frac{M R S_{N_{\pi^{h}}{ }} N_{s}+\pi_{s}^{h}}{M R S_{N_{\pi^{h}}} N_{s}} \frac{\pi_{q}^{p}}{\pi_{s}^{p}}=\frac{N_{q}}{N_{s}}
\end{aligned}
$$

With the exception of the first term on the left hand side (LHS), (23) is identical to (13). This (positive) term determines the difference between the equilibrium $q$ and $s$ under the full-cost payment scheme and the prospective payment scheme. Again, the relationship between the slopes of the indifference curve and the iso-profit depends on whether services are substitutes or complements. For substitutes, the coefficient of the iso-profit's slope is less than one. Thus, (23) shows that, under the prospective payment scheme, the equilibrium $q$ and $s$ occurs at a point where the iso-profit is steeper than the indifference curve but not as steep as under the full-cost scheme. This is shown in Figure 3(a) as (ii) which has less $q$ and more $s$ than the full cost equilibrium ${ }^{7}$. This result is equivalent to [16]. When services are complements, the coefficient of the iso-profit's slope in (23) is greater than one implying that in equilibrium the isoprofit is flatter than the indifference curve but not as flat as under the full cost. As shown in Figure 3(b) as ii, the equilibrium $q$ and $s$ are both less than those of the full cost and for a given level of private profit, the patient ends up on a lower indifference curve than the full cost equilibrium. This again is consistent with the results of [16].

To determine the efficiency of this equilibrium (2), (3), (6), (8), and (9) are substituted into (23) set $B_{s}=c^{p}$ (i.e., efficiency in the private sector), and rearranged to produce:

$$
k=\left(1-M R S_{N_{\pi^{h}}}\right)\left[\alpha B_{q}+q(1-\alpha) \frac{B_{s q}^{2}-B_{q q} B_{s s}}{B_{s s}}\right]
$$

where $k=B_{q}-c^{h}=0$ is required for efficiency in the public sector. Eq.24 shows that $k=0$ when $M R S_{N \pi}^{h}=1$. However, $k>0$ when $M R S_{N \pi}^{h}<1$ and $k<0$ when $M R S_{N \pi}^{h}>1^{8}$. Efficiency in the public sector depends 


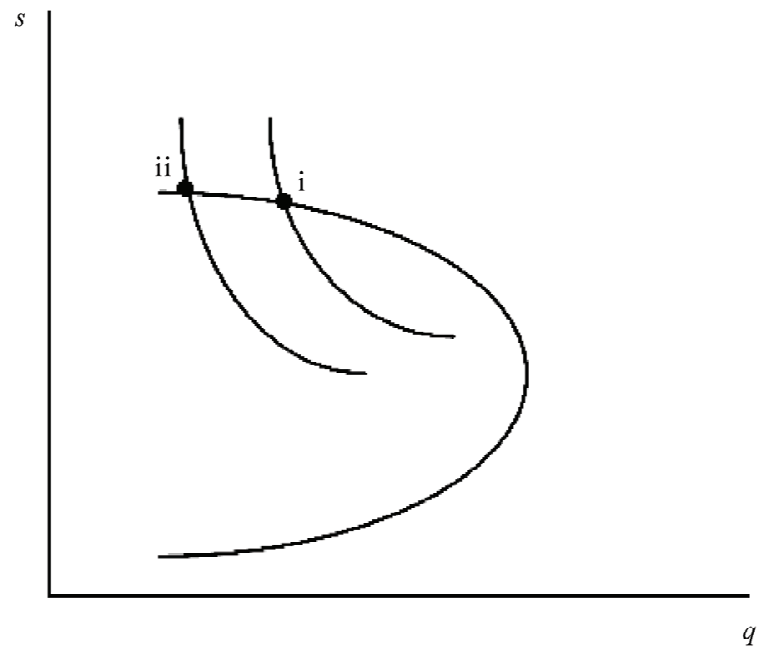

(a)

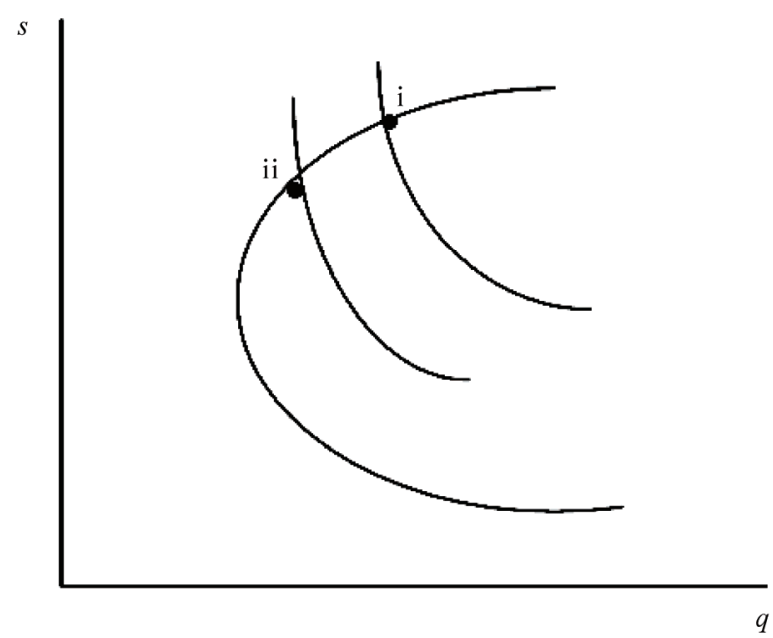

(b)

Figure 3. (a) Prospective payment equilibrium for substitutes relative to that of full cost; (b) Prospective payment equilibrium for complements relative to that of full cost.

on the agency behaviour of the physician and not on the relationship between the services. Perfect agency is required for efficiency in the public sector while imperfect agency produces too little $q$ in equilibrium. Given that the physician is an imperfect agent, (24) shows that term in the square bracket increases when the price elasticity of demand for $s$ increases ${ }^{9}$. As the demand for services in the private sector becomes more elastic the imperfect agent supplies an optimal amount of private services at

${ }^{9}$ Note that $\varepsilon_{s}=\frac{B_{s}}{s B_{s s}} \Rightarrow B_{s s}=\frac{B_{s}}{s \varepsilon_{s}}$, substituting this into (24) and differ-

entiating with respect to $\varepsilon_{\mathrm{s}}$ yields $s q(1-\alpha) \frac{B_{s q}^{2}}{B_{s}}>0$. the expense of too little supply of public services regardless of the relationship between the services.

These results are also important. First, compared with the cost reimbursement case, the disincentive that accompanies the provision of $q$ under the prospective payment causes the imperfect agent to cut down the oversupply of $q$ that exist under the cost reimbursement when services are complements as well as the optimal supply of $q$ when services are substitutes such that too little $q$ is produced whether services are complements or substitutes. Second, the results here are contrary to [16] where efficiency in both sectors is possible under the prospective payment when services are substitutes regardless of the agency type of the physician. Third, the results are similar to [15] in that perfect agency is required for efficiency. Reference [15] argues that the need for perfect agency weakens the argument in favour of prospective payment scheme. The influence of hospitals on physicians' behaviour is manifested in the change in services provided by physicians in accordance with changes in the payment scheme to hospitals. Thus, physicians are more likely to be imperfect agents than perfect agents implying that efficiency cannot be actualized under the prospective payment. The results also provide interesting comparison with [10], [16] and [12]. Reference [10] presents a model in which all firms (hospitals) produce homogeneous product but differ by the cost of production. He concludes that prospective payment, because it makes payment independent of the hospital's cost, is optimal. Reference [16] considers a model with heterogeneous patients, in terms of costliness, that can be treated with varying efforts with demand responding to the variation. Managerial effort is required for the enhancement of quality and reduction of cost. His results show that prospective payment can elicit the efficient effort if the provider has to treat all patients. Selden's results, however, showed that prospective payment is not optimal even under full insurance.

The question then is what size of $\alpha$ can lead to efficiency in the public sector, under the prospective payment, given efficiency in the private sector. This is found from (24) by setting $k=0$ and solving for $\alpha$ :

$$
\alpha^{*}=\frac{q\left(B_{s q}^{2}-B_{s s} B_{q q}\right)}{q\left(B_{s q}^{2}-B_{s s} B_{q q}\right)-B_{q} B_{s s}}
$$

Thus, there is an optimal $\alpha$ at which efficiency in both sectors is possible under the prospective payment regardless of the type of agency role played by the physician as well as the relationship between services. Obviously, $\alpha^{*}<1$. Note, however, that $\alpha^{*}$ increases as the elasticity of demand for private service falls regardless of the relationship between the private and public ser- 
vices $^{10}$. This is interesting because (24) shows that given that the physician is imperfect, a fall in the elasticity of demand for private services causes the physician to increase services in the public sector given an optimal supply of services in the private sector. Thus, (25) implies that as the services in the private and/or the public sector become necessities (and so less elastic) efficiency demands that the government reduces the user fee for public services. This is consistent with [16] that when $\alpha$ $=1$ efficiency is possible under prospective payment regardless of the type of agency.

\subsection{Cost Sharing}

Under this rule, the government makes a fixed payment, $\mathrm{G}$, and covers a fraction, $\gamma$, of the cost of production:

$$
R(q)=G+\gamma c(q)
$$

The public hospital profit becomes:

$$
\pi^{h}=G+(1-\alpha) B_{q}(q, s) q+(\gamma-1) c(q)
$$

where $0<\gamma<1$. The government can increase, $G$, and reduce $\gamma$ such that the total payment remains constant. Note that $\gamma=0$ implies prospective payment while $G=0$ and $\gamma=1$ represents full cost reimbursement. The marginal profit is:

$$
\pi_{q}^{h}=(1-\alpha) B_{q}(q, s)+q(1-\alpha) B_{q q}(q, s)-(1-\gamma) c^{\prime}(q)<0
$$

Eq.13 becomes:

$$
\begin{aligned}
& \frac{(1-\gamma) c^{\prime}(q)-q(1-\alpha) B_{q q}(q, s)-(1-\alpha) B_{q}(q, s)}{M R S_{N_{\pi^{h}}} N_{s}} \\
& +\frac{M R S_{N_{\pi^{h}}} N_{s}+\pi_{s}^{h}}{M R S_{N_{\pi^{h}}} N_{s}} \frac{\pi_{q}^{p}}{\pi_{s}^{p}}=\frac{N_{q}}{N_{s}}
\end{aligned}
$$

With the exception of - $\gamma c^{\prime}(q),(29)$ is identical to (23). Eq.29 shows that for substitutes the iso-profit is steeper than the patient's indifference curve but not as steep as under the prospective payment. When services are complements, however, the iso-profit is flatter than the patient's indifference curves but by lower degree than under the prospective payment. These are shown as iii in Figures 4(a) and 4(b):

Thus, patients are worse off under cost sharing than under full cost but are not as worse off as under prospective payment.

Following [16], it is important to consider the size of $\gamma$

${ }^{10}$ The elasticity of demand for $s$ is: $\mathcal{E}_{s}=\frac{B_{s}}{s B_{s s}} \Rightarrow B_{s s}=\frac{B_{s}}{s \mathcal{E}_{s}}$;

$\frac{\partial \alpha^{*}}{\partial B_{s s}}=\frac{q B_{q} B_{s q}^{2}}{\left[q\left(B_{s q}^{2}-B_{s s} B_{q q}\right)-B_{q} B_{s s}\right]^{2}}>0$.

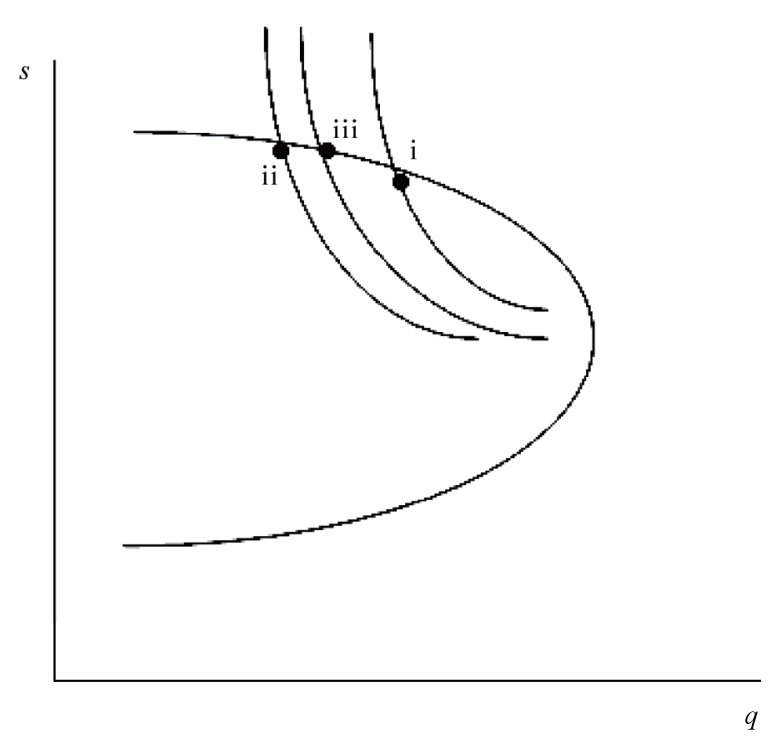

(a)

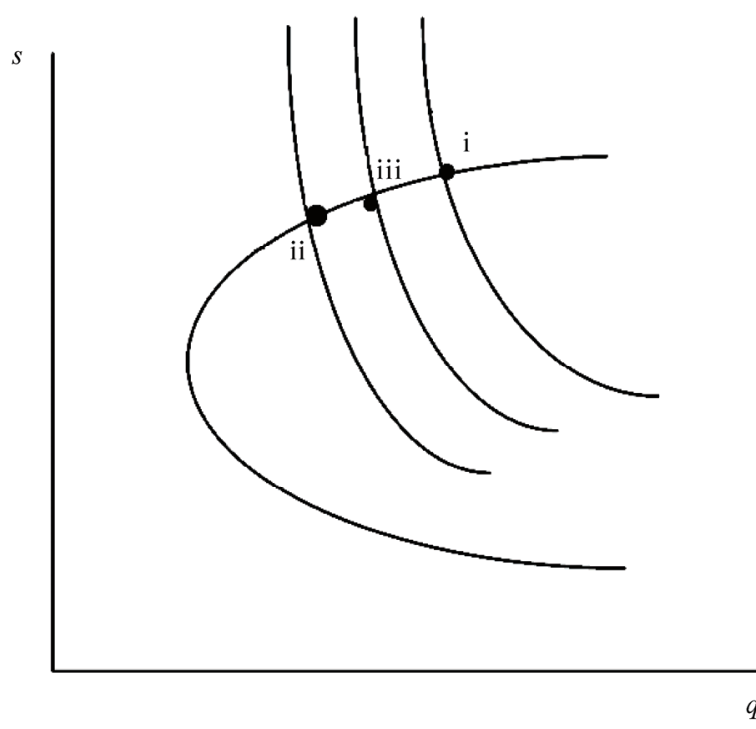

(b)

Figure 4. (a) Cost sharing equilibrium for substitutes, relative to those of full cost and prospective payment; (b) Cost sharing equilibrium for complements relative to those of full cost and prospective payment.

at which optimality can be achieved in the public sector given that the private sector is optimal. This is obtained by substituting (2), (3), (6), (8) and (9) into (29) and setting $k=0$ :

$$
\gamma^{*}=\left(1-M R S_{N_{\pi^{h}}}\right)\left(\alpha+\frac{q(1-\alpha)\left(B_{s q}^{2}-B_{s s} B_{q q}\right)}{c^{\prime}(q) B_{s s}}\right)
$$

Clearly $\gamma^{*}$ is zero when the physician is a perfect agent in which case prospective payment is optimal. 
However, $\gamma^{*}$ cannot be one ${ }^{11}$, i.e., cost sharing can be used to ensure optimal supply of services. It is optimal for the government to use a combination of fixed fee and incentive payment to reward the hospital because this forces providers to internalize the externalities that leads to undersupply under prospective payment (Selden 1990; [15] 1986) and oversupply under full cost reimbursement. Equation (30) shows that the size of $\gamma^{*}$ does not depend on the relationship between services i.e., it is optimal for the government to pass on the same fraction of cost for substitutes and complements. In [16] efficiency in both sectors requires that $\gamma^{*}=\left(1-M R S_{N \pi}{ }^{h}\right)$. This is identical to what [15] recommend for efficiency in the public sector when there is no private sector. Thus, in Rickman \& McGuire, the optimal fraction of cost that is passed on to providers does not depend on the relationship between the services.

The term in the square bracket in (30) represents the effect of the user fee in the public sector on the size of $\gamma^{*}$. Note that when $\alpha=1$ (30) becomes $\gamma^{*}=\left(1-M R S_{N \pi}{ }^{h}\right)$, which is the same as in [16] as well as in [15]. With the term in the square bracket less than one, $\gamma^{*}$ in the current model is less than that in [16]. Thus, when there is a user fee in the public sector, efficiency requires that less revenue be retained to induce optimal provision of public services for both substitutes and complements than when there is no user fee. Note that the second term in the square bracket increases in the elasticity of services in both sectors ${ }^{12}$. The $\gamma^{*}$ here is subject to the same setback as in [15] as well as [16] in that information on an unobservable variable, the physician's utility, is required. However, the effect of elasticity on $\gamma^{*}$ in the current model reduces the dependence on information on the physician's utility. The presence of the positive term in the bracket which depends on elasticity reduces the range within which $\gamma^{*}$ falls. Thus, under the user fee system in the public sector in a two-tier system, cost sharing can ensure efficiency in both the public and private sector regardless of the relationship between services in the two sectors. This is also intuitive. Compared to the full cost reimbursement system (where $G=0$ and $\gamma=1)$, the fall in marginal revenue resulting from letting $\gamma^{*}<1$ gives the imperfect agent the incentive to reduce the oversupply of complementary services while maintaining supply of substitutes at the efficient level. For comparison with prospective payment, (where $G>0$ and $\gamma=0$ ) the increase in marginal revenue from setting $\gamma^{*}<$ 1 induces the imperfect agent to increase services.

${ }^{11}$ This is based on the assumption that $\frac{q\left(B_{s q}^{2}-B_{s s} B_{q q}\right)}{c^{\prime}(q) B_{s s}}<1$
${ }^{12} \frac{\partial()}{\partial B_{s s}}=-\frac{q(1-\alpha) B_{s q}^{2}}{B_{s s}^{2}}<0$ and $\quad \frac{\partial()}{\partial B_{q q}}=-\frac{q(1-\alpha)}{c^{\prime}(q)}<0$

\section{CONCLUSIONS}

Many recent health care reforms introduce user fees to the public sector. This paper examined patient's welfare and efficiency under different provider reimbursement schemes in the public sector in a mixed health care system where the patient bears cost for treatment in both the public and private sectors. The paper extended previous studies by introducing user fee in the public sector. The provider reimbursement schemes examined are full-cost reimbursement, prospective payment and cost sharing.

The results show that efficiency is possible under the full cost reimbursement scheme if the physician trades off public hospital surplus for patient net benefit when services are substitutes and trading off patient's net benefit for public hospital surplus or being a perfect agent when services are complements. This is contrary to the results in previous studies where there is oversupply of services in equilibrium in the public sector under the full cost reimbursement. Under the prospective payment efficiency is only possible when the fraction of cost not covered by the user fee is at its optimal level. Similarly, under the cost-sharing scheme, efficiency occurs only if the fraction of cost that the government passes on to providers is at its optimal level. This is similar to the results in previous studies; however, the optimal fraction in this paper is less than that in previous studies. Of the three reimbursements schemes, the patient is worst off under the prospective payment and has the highest utility under the full-cost reimbursement. In general the introduction of user fee in the public sector makes the patient worse off when services are substitutes. However, it is not clear whether the user fee makes patients worse off or better off when services are complements.

\section{REFERENCES}

[1] Besley, T. and Gouveia, B. (1994) Alternative systems of health care provision. Economic Policy, 203-258.

[2] Zweifel, P. and Breyer, F. (1997) Health economics. Oxford University Press, Oxford.

[3] Petretto, A. (1999) Optimal social health insurance with supplementary private insurance. Journal of Health Economics, 18(1), 727-745.

[4] Holmer, M. (1984) Tax policy and the demand for health insurance. Journal of Health Economics, 3, 203-221.

[5] Zuckerman, S. (1987) Commercial insurers and all-payer regulation evidence on hospitals' responses to financial need. Journal of Health Economics, 6(3), 165-187.

[6] Gravelle, H. (1999) Capitation contracts: access and quality. Journal of Health Economics, 18(1), 315-340.

[7] Chernick, H.A., Holmer, M.R. and Weinberg, D.H. (1987) Tax policy toward health insurance and the demand for medical services. Journal of Health Economics, 61-25.

[8] Selden, T.M. (1999) Premium subsidies for health insur- 
ance: Excessive coverage vs. adverse selection. Journal of Health Economics, 18, 709-725.

[9] Pope, G. (1990) Hospital nonprice competition and medicare reimbursement policy. Journal of Health Economics, 8(2), 147-172

[10] Schleifer, A. (1985) A theory of yardstick competition. The RAND Journal of Economics, 16(3), 319-327.

[11] Keeler, E.B. (1990) What proportion of hospital cost differences is justifiable? Journal of Health Economics 9(3), 359-365.

[12] Selden, T.M. (1990) A model of capitation. Journal of Health Economics, 9, 397-409.

[13] Ellis, R.P. and McGuire, T.G., (1993) Supply-side and demand-side cost sharing in health care. Journal of Economic Perspectives, 7(4), 135-151.

[14] Ma, C. A. (1994) Health care payment systems: Cost and quality incentives. Journal of Economics \& Management Strategy, 3(1), 93-112.

[15] Ellis, R.P., McGuire, T.G., (1986) Provider behavior under prospective reimbursement, cost sharing and supply, Journal of Health Economics, 5(1), 129-151.

[16] McGuire, A. and Rickman, N. (1999) Regulating provider's reimbursement in a mixed market for health care. Scottish Journal of Political Economy, 46(1), 53-71.

[17] Leger, P.T. (2000) Quality control mechanisms under capitation payment for medical services. Canadian Journal of Economics, 33(2), 565-586.

[18] Leonard, K.L. (1998) Institutional structure of health care in rural cameroun: Structural estimation of produc- tion in teams with unobservable effort. Discussion Paper Series N, 9798-9816.

[19] Bloom, G. and Segall, M. (1994) Expenditure and financing of the health sector inkenya, abridged report of a study performed for the ministry of health and the world bank. IDS Commissioned Study, 9.

[20] Lavy, V. and Germain, J. (1995) Tradeoffs in cost, quality and accessibility in utilization of health facilities: Insights from Ghana. In: Shaw, R.P. and Ainsworth, Eds., Financing Health Services through User Fees and Insurance Case Studies from Sub-Saharan Africa, World Bank Discussion Papers, 103-121.

[21] Petretto, A. (1999) Optimal social health insurance with supplementary private insurance. Journal of Health Economics, 18(3), 727-745.

[22] Evans, R.G. (1974) Supplier-induced demand: Some empirical evidence and implications. In: Perlman, M., Ed., The Economics of Health and Medical Care, Wiley, New York.

[23] Farley, P. (1986) Theories of the price and quantity of physician services: A synthesis and critique. Journal of Health Economics, 5(1), 315-333.

[24] Laffont, J.J. and Tirole, J. (1986) Optimal payment systems for health services. Journal of Health Economics, 9(4), 375-396.

[25] Laffont, J.-J. and Tirole, J. (1993) A theory of incentives in procurement and regulation. MIT Press, Cambridge. 